\title{
Port-Hamiltonian Modeling of Systems with Position-Dependent Mass *
}

\author{
Dimitri Jeltsema* Arnau Dòria-Cerezo** \\ * Delft Institute of Applied Mathematics, Delft University of \\ Technology, Mekelweg 4, 2628 CD Delft, The Netherlands \\ (email: d.jeltsema@tudelft.nl) \\ ** Dept. of Electrical Engineering and Institute of Industrial and \\ Control Engineering, Universitat Politècnica de Catalunya, Av. Víctor \\ Balaguer s/n, 08800 Vilanova i la Geltrú, Spain \\ (email: arnau.doria@upc.edu)
}

\begin{abstract}
It is known that straightforward application of the classical Lagrangian and Hamiltonian formalism to systems with mass varying explicitly with position may lead to discrepancies in the formulation of the equations of motion. Systems with mass varying explicitly with position often arise from situations where the partitioning of a closed system of constant mass leads to open subsystems that exchange mass among themselves. One possible solution is to introduce additional non-conservative generalized forces that account for these effects. However, it remains unclear how to systematically interconnect the Lagrangian or Hamiltonian subsystems. In this paper, systems with mass varying explicitly with position and their properties are studied in the port-Hamiltonian modeling framework. The portHamiltonian formalism combines the classical Lagrangian and Hamiltonian approach with network modeling and is applicable to various engineering domains. One of the strong aspects of the port-Hamiltonian formalism is that power-preserving interconnections between portHamiltonian subsystems results in another port-Hamiltonian system with composite energy and interconnection structure. The motion of a heavy cable being deployed from a reel by the action of gravity is used as an example.
\end{abstract}

Keywords: Modeling, variable mass systems, classical mechanics, port-Hamiltonian systems, open systems.

\section{INTRODUCTION}

A prevailing trend in the description of physical systems for analysis, control applications, and simulation is network modeling. The system is split into open subsystems (tearing), the subsystems are modeled (zooming), and the model of the overall system is obtained by interconnecting the models of the subsystems (linking) (Willems (2007)). The main advantage of such approach is that the models of the physical subsystems can be stored in libraries. The modeling process can be performed in an iterative manner and the model of the overall system is simply constructed by interconnecting the library sub-models.

Although network modeling has proven a powerful framework for many engineering problems, special care should be devoted to systems in which mass is transported from one subsystem to another. Apart from the classical rocket problem or the problem of a chain being coiled up at a table, some interesting examples of such systems can be found in the textile industry (Cveticanin (1998)) or devices in which cables are deployed or retrieved, such as tethered satellites (Crelling et al. (1997)) and lifting cranes in off-shore engineering (Pesce et al. (2006)). From

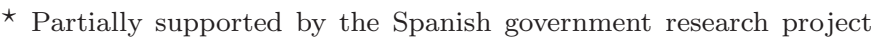
DPI2010-15110.
}

a closed system perspective the mass of these systems may considered to be constant, but the decomposition into open subsystems will lead to systems with variable mass. The derivation of the equations of motion for systems with variable mass is not as straightforward as it would normally be for a system with constant mass. The reason is that the basic principles of dynamics, such as Lagrange's or Hamilton's equations, are only truly valid when applied to a definite set of particles or rigid bodies. Indeed, in (Pesce (2003)) it is shown that a naive application of the usual Lagrange equations to a system with mass varying explicitly with position generally leads to erroneous equations of motion. Hence, to study systems with varying mass, one can proceed by extending the Lagrange equations. In a fashion similar as the inclusion of dissipative forces via the usual Rayleigh dissipation function, such approach basically consists in adding terms to the righthand side of the equation that compensate for the nonconservative forces stemming from the loss or gained mass in the system. However, the addition of non-conservative forces destroys the variational character of the equations, i.e., the system is not completely characterized by its Lagrangian alone. Another problem that arises is how the various subsystems can be interconnected in a systematic and consistent manner. 
In this paper, we study physical systems with mass varying explicitly with position and their properties in the portHamiltonian modeling framework. The port-Hamiltonian formalism combines the classical Lagrangian and Hamiltonian approach with network modeling in a variety of domains (e.g., mechanical, electrical, electromechanical, hydrodynamical, and thermodynamical); see (Van der Schaft (2000)) for a basic introduction and (Duindam et al (2009)) for a comprehensive summary of the developments of this framework over the past decade. Exposing the relation between the energy storage, dissipation, and interconnection structure, this framework underscores the physics of the system. One of the strong aspects of the portHamiltonian formalism is that a power-preserving interconnection between port-Hamiltonian systems results in another port-Hamiltonian system with composite energy, dissipation, and interconnection structure. Based on this principle, complex, multidomain systems can be modeled by interconnecting port-Hamiltonian descriptions of its subsystems.

In order to present the essential ideas as clear as possible we will treat the simple problem of a heavy cable being deployed from a reel by the action of gravity. Section 2 briefly reviews the application of the Lagrangian and Hamiltonian formalism from both a closed and open system perspective. The discrepancies occurring in the latter case are highlighted and possible solutions are discussed. In Section 3 , it is first briefly shown how the classical Hamiltonian equations can be generalized to port-Hamiltonian systems with dissipation. Next, the port-Hamiltonian structure is accommodated to include systems with mass depending explicitly on position. The paper is concluded by considering the cable-reel system as the interconnection of two open port-Hamiltonian (sub)systems in Section 4, and some final remarks in Section 5.

\section{BACKGROUND MATERIAL AND A MOTIVATING EXAMPLE}

In classical mechanics the starting point in setting up the Lagrangian equations of motion is to determine the Lagrangian, see e.g., (Abraham and Marsden (1978)). For a mechanical system consisting of $N$ particles with constant mass $m_{k}, k=1,2, \ldots, N$, the Lagrangian is given by $L(\boldsymbol{r}, \dot{\boldsymbol{r}})=T^{*}(\dot{\boldsymbol{r}})-V(\boldsymbol{r})$, where $\boldsymbol{r}=\operatorname{col}\left(\boldsymbol{r}_{1}, \ldots, \boldsymbol{r}_{N}\right)$ represents the positions of the particles, $\dot{\boldsymbol{r}}=\mathrm{d} \boldsymbol{r} / \mathrm{d} t$ are the corresponding velocities, $T^{*}(\dot{\boldsymbol{r}})$ is the kinetic (co-)energy

$$
T^{*}(\dot{\boldsymbol{r}})=\frac{1}{2} \sum_{k=1}^{N} m_{k}\left\|\dot{\boldsymbol{r}}_{k}\right\|^{2},
$$

and $V(\boldsymbol{r})$ is the potential energy. If the system is subjected to holonomic constraints, then it is convenient to write the Lagrangian in terms of generalized coordinates $\boldsymbol{q}=$ $\operatorname{col}\left(q_{1}, \ldots, q_{n}\right)$, where $n \leq 3 N$ is the number of degrees of freedom. Letting $\boldsymbol{r}=\hat{\boldsymbol{r}}(\boldsymbol{q})$, then the kinetic (co-)energy takes the form

$$
T^{*}(\boldsymbol{q}, \dot{\boldsymbol{q}})=\frac{1}{2} \dot{\boldsymbol{q}}^{T} \boldsymbol{M}(\boldsymbol{q}) \dot{\boldsymbol{q}},
$$

with generalized mass matrix

$$
\boldsymbol{M}(\boldsymbol{q}):=\left[\frac{\partial \hat{\boldsymbol{r}}}{\partial \boldsymbol{q}}(\boldsymbol{q})\right]^{T}\left[\begin{array}{lll}
m_{1} & & \\
& \ddots & \\
& & m_{k}
\end{array}\right] \frac{\partial \hat{\boldsymbol{r}}}{\partial \boldsymbol{q}}(\boldsymbol{q}) .
$$

The equations of motion, which are equivalent to Newton's second law, are then the Lagrange equations

$$
\frac{\mathrm{d}}{\mathrm{d} t} \frac{\partial L}{\partial \dot{q}_{j}}-\frac{\partial L}{\partial q_{j}}=0, \quad j=1,2, \ldots, n,
$$

where $L(\boldsymbol{q}, \dot{\boldsymbol{q}})=T^{*}(\boldsymbol{q}, \dot{\boldsymbol{q}})-V(\boldsymbol{q})$.

In passing on to the Hamiltonian formulation, we define the conjugate momenta $\boldsymbol{p}=\operatorname{col}\left(p_{1}, \ldots, p_{n}\right)$ as $p_{j}=\frac{\partial L}{\partial \dot{q}_{j}}$, which in this case are simply given by $\boldsymbol{p}=\boldsymbol{M}(\boldsymbol{q}) \dot{\boldsymbol{q}}$. Hence, the second-order equations (4) transform into $2 n$ first order equations

$$
\begin{aligned}
\dot{q}_{j} & =\frac{\partial H}{\partial p_{j}}, \\
\dot{p}_{j} & =-\frac{\partial H}{\partial q_{j}}, \quad j=1,2, \ldots, n,
\end{aligned}
$$

where the Hamiltonian

$$
H(\boldsymbol{q}, \boldsymbol{p})=\frac{1}{2} \boldsymbol{p}^{T} \boldsymbol{M}^{-1}(\boldsymbol{q}) \boldsymbol{p}+V(\boldsymbol{q})
$$

represents the total energy stored by the system. A key property of (5) is that the rate of change of the Hamiltonian

$$
\dot{H}(\boldsymbol{q}, \boldsymbol{p})=0,
$$

expressing that the energy is conserved within the system.

The Lagrange equations (4), with as Lagrangian the difference between the kinetic co-energy and the potential energy, are only valid for conservative systems. The same holds for the associated Hamiltonian equations (5). If the system is not purely conservative it can not completely be characterized by its Lagrangian. Moreover, as will be shown next, a straightforward application of the usual Lagrange equations to a system with mass varying explicitly with position leads to discrepancies in the formulation of the equations of motion.

\subsection{Cable-Reel System: A Closed System Approach}

Consider the elementary problem of a heavy cable that is deployed from a reel depicted in Fig. 1 by the action of gravity. The empty reel has radius $R$ and moment of inertia $I_{o}$ around the axis of rotation. The mass per unit length of the non-stretching and infinitely flexible cable is denoted by $\mu$. For simplicity we assume that the diameter of the cable is very small compared to the diameter of the reel and that all turns can be accommodated into a single winding layer. Furthermore, let $\ell$ be the total length of the cable such that the total mass of the cable equals $m_{c}=\mu \ell$. If $q_{r}$, with $q_{r} \leq \ell$, represents the length of the cable that is wounded on the reel, then the cable's wounded and suspended mass equal $m_{r}=\mu q_{r}$ and $m_{s}=m_{c}-m_{r}$, respectively.

As shown in (Pesce (2003)), when the cable-reel system is considered as a closed (invariant mass) system, the equation of motion can be derived using the Lagrangian formalism. Indeed, selecting the angular displacement $\theta_{r}$ as the configuration coordinate, together with its associated angular velocity $\omega_{r}=\dot{\theta}_{r}$, the total kinetic (co-)energy is given by

$$
\begin{aligned}
T^{*}\left(\omega_{r}\right) & =\frac{1}{2}\left(I_{o}+m_{r} R^{2}\right) \omega_{r}^{2}+\frac{1}{2} m_{s} \dot{q}_{s}^{2} \\
& =\frac{1}{2}\left(I_{o}+m_{c} R^{2}\right) \omega_{r}^{2},
\end{aligned}
$$




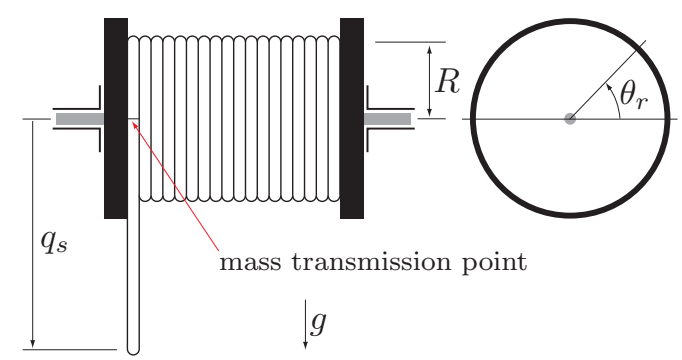

Fig. 1. A cable-reel system.

where we have used the fact that translational displacement can be expressed as $q_{s}=\ell-R \theta_{r}$, so that the translational velocity equals $\dot{q}_{s}=-R \omega_{r}$, respectively. The potential energy due to the action of gravity is given by

$$
V\left(\theta_{r}\right)=-\int_{0}^{\ell-R \theta_{r}} g m_{s} \mathrm{~d} q_{s}=-\frac{1}{2} g \mu\left(\ell-R \theta_{r}\right)^{2} .
$$

Hence, defining the Lagrangian $L\left(\theta_{r}, \omega_{r}\right)=T^{*}\left(\omega_{r}\right)-V\left(\theta_{r}\right)$ and evaluating the usual Lagrange equation

$$
\frac{\mathrm{d}}{\mathrm{d} t}\left(\frac{\partial L}{\partial \omega_{r}}\right)-\frac{\partial L}{\partial \theta_{r}}=0
$$

yields $\left(I_{o}+m_{c} R^{2}\right) \dot{\omega}_{r}+g \mu R\left(\ell-R \theta_{r}\right)=0$.

\subsection{Cable-Reel System: An Open System Perspective}

Physically the cable-reel system can be separated into two subsystems at the mass transmission port indicated in Fig. 1. At this port, the reel and the wounded part of the cable gain mass at rate $\dot{m}_{r}=\mu R \omega_{r}$, whereas the suspended part of cable loses mass at rate $\dot{m}_{s}=-\mu \dot{q}_{s}$, and vice-versa. Let us first consider the subsystem formed by the reel and the wound part of the cable. Intuitively, it seems natural to consider this subsystem as just a position-dependent inertia $I_{r}\left(\theta_{r}\right)=I_{o}+\mu R^{3} \theta_{r}$, with $\theta_{r} \geq$ 0 . Since a moment of inertia is defined by a relationship between angular momentum $p_{r}$ and angular velocity $\omega_{r}$, we have $p_{r}=I\left(\theta_{r}\right) \omega_{r}$. Hence, the equation of motion for this subsystem is given by

$$
\dot{p}_{r}=\left(I_{o}+\mu R^{3} \theta_{r}\right) \dot{\omega}_{r}+\mu R^{3} \omega_{r}^{2}=\tau_{r},
$$

where $\tau_{r}$ denotes some external torque acting on the subsystem. In deriving the equation of motion using the Lagrangian approach, we define $L_{r}\left(\theta_{r}, \omega_{r}\right)=\frac{1}{2} I_{r}\left(\theta_{r}\right) \omega_{r}^{2}$, and evaluate

$$
\frac{\mathrm{d}}{\mathrm{d} t}\left(\frac{\partial L_{r}}{\partial \omega_{r}}\right)-\frac{\partial L_{r}}{\partial \theta_{r}}=\tau_{r}
$$

However, we now obtain

$$
\left(I_{o}+\mu R^{3} \theta_{r}\right) \dot{\omega}_{r}+\frac{1}{2} \mu R^{3} \omega_{r}^{2}=\tau_{r},
$$

which, in comparison to (10), contains an erroneous factor $\frac{1}{2}$.

A similar discrepancy occurs using the Hamiltonian equations. Indeed, let

$$
H_{r}\left(\theta_{r}, p_{r}\right)=\frac{1}{2} I_{r}^{-1}\left(\theta_{r}\right) p_{r}^{2},
$$

yielding

$$
\begin{aligned}
& \dot{\theta}_{r}=\frac{\partial H_{r}}{\partial p_{r}}=\frac{p_{r}}{I_{o}+\mu R^{3} \theta_{r}} \\
& \dot{p}_{r}=-\frac{\partial H_{r}}{\partial \theta_{r}}+\tau_{r}=\frac{1}{2} \mu R^{3}\left(\frac{p_{r}}{I_{o}+\mu R^{3} \theta_{r}}\right)^{2}+\tau_{r},
\end{aligned}
$$

while we know from (10) that $\dot{p}_{r}=\tau_{r}$.

The main reason for this discrepancy is due to the fact that the Lagrange and Hamiltonian equations in the form of (11) and (14), respectively, are generally only suited for conservative systems. Since the reel is gaining or losing mass via the mass transmission port, it is not conservative or lossless. Starting from Newton's second law, we have

$$
\omega_{r} \frac{\mathrm{d}}{\mathrm{d} t}\left(I_{r}\left(\theta_{r}\right) \omega_{r}\right)=I_{r}\left(\theta_{r}\right) \omega_{r} \dot{\omega}_{r}+\dot{I}_{r}\left(\theta_{r}\right) \omega_{r}^{2}=\omega_{r} \tau_{r},
$$

which, by using $\mathrm{d}\left(\frac{1}{2} I_{r}\left(\theta_{r}\right) \omega_{r}^{2}\right) / \mathrm{d} t=I_{r}\left(\theta_{r}\right) \omega_{r} \dot{\omega}_{r}+\frac{1}{2} \dot{I}_{r}\left(\theta_{r}\right) \omega_{r}^{2}$, can be written as

$$
\frac{\mathrm{d}}{\mathrm{d} t}\left(\frac{1}{2} I_{r}\left(\theta_{r}\right) \omega_{r}^{2}\right)=\omega_{r} \tau_{r}-\frac{1}{2} \dot{I}_{r}\left(\theta_{r}\right) \omega_{r}^{2} .
$$

Integrating the latter over the interval $\left[t_{0}, t_{1}\right]$ yields

$$
\begin{gathered}
\frac{1}{2} I_{r}\left(\theta_{r}\left(t_{1}\right)\right) \omega_{r}^{2}\left(t_{1}\right)-\frac{1}{2} I_{r}\left(\theta_{r}\left(t_{0}\right)\right) \omega_{r}^{2}\left(t_{0}\right)= \\
\int_{t_{0}}^{t_{1}} \omega_{r}(t) \tau_{r}(t) \mathrm{d} t-\frac{1}{2} \int_{t_{0}}^{t_{1}} \dot{I}_{r}\left(\theta_{r}(t)\right) \omega_{r}^{2}(t) \mathrm{d} t,
\end{gathered}
$$

which states that the stored energy equals the supplied energy minus the loss or gain of energy due to the outflow or inflow of mass, respectively.

A possible solution to compensate for the erroneous term is to add to the right-hand sides of (11) and (14) an additional term $-\frac{1}{2}\left(\mathrm{~d} I_{r}\left(\theta_{r}\right) / \mathrm{d} \theta_{r}\right) \omega_{r}^{2}$; see (Pesce $\left.(2003)\right)$. However, two problems arise: (i) the variational character that makes the Lagrangian equations so appealing is lost, and (ii) if a similar Lagrangian subsystem for the suspended part of the cable is derived it is unclear how to formally describe the interconnection between the two subsystems.

\section{THE PORT-HAMILTONIAN FORMALISM}

Historically, the Hamiltonian approach has its roots in analytical mechanics and starts from the principle of least action, via the Lagrange equations and the Legendre transformation, towards the Hamiltonian equations of motion. On the other hand, the network modeling approach stems from electrical engineering, and constitutes a cornerstone of systems theory. While much of the analysis of physical systems has been performed within the Lagrangian and Hamiltonian framework, the network modeling point of view is prevailing in modeling and simulation of (complex) physical systems. The framework of port-Hamiltonian systems combines both points of view. Although the general structure of a port-Hamiltonian system possesses very rich mathematical properties, we will only highlight its most employed form that is suitable for the problem at hand. The interested reader is referred to (Van der Schaft (2000)) for a basic introduction, and to (Duindam et al. (2009)) for a comprehensive summary of the developments of this framework over the past decade. 


\subsection{Input-State-Ouput Hamiltonian Systems}

A major generalization of the classical Hamiltonian formalism is given by the port-Hamiltonian equations

$$
\begin{aligned}
& \dot{\boldsymbol{x}}=\boldsymbol{J}(\boldsymbol{x}) \frac{\partial H}{\partial \boldsymbol{x}}(\boldsymbol{x})+\boldsymbol{G}(\boldsymbol{x}) \boldsymbol{u}, \\
& \boldsymbol{y}=\boldsymbol{G}^{T}(\boldsymbol{x}) \frac{\partial H}{\partial \boldsymbol{x}}(\boldsymbol{x}),
\end{aligned}
$$

where $\boldsymbol{x}$ are local coordinates for an $n$-dimensional state space manifold $\mathcal{X}$, the Hamiltonian $H(\boldsymbol{x})$ denotes the total stored energy, $\boldsymbol{u}, \boldsymbol{y} \in \mathbb{R}^{m}$ denote the system's port variables (i.e., inputs and outputs, respectively), $\boldsymbol{G}(\boldsymbol{x})$ denotes the $n \times m$ port distribution matrix, and $\boldsymbol{J}(\boldsymbol{x})$ is an $n \times n$ matrix, which is assumed to be skew-symmetric, i.e., $\boldsymbol{J}(\boldsymbol{x})=-\boldsymbol{J}^{T}(\boldsymbol{x})$. Because of skew-symmetry of $\boldsymbol{J}(\boldsymbol{x})$, it is easily noted that $\dot{H}(\boldsymbol{x})=\boldsymbol{u}^{T} \boldsymbol{y}$, which upon integration over the time interval $\left[t_{0}, t_{1}\right]$, i.e.,

$$
H\left(\boldsymbol{x}\left(t_{1}\right)\right)-H\left(\boldsymbol{x}\left(t_{0}\right)\right)=\int_{t_{0}}^{t_{1}} \boldsymbol{u}^{T}(t) \boldsymbol{y}(t) \mathrm{d} t,
$$

expresses the fact that the increase of internal energy in the system is equal to the externally supplied work (conservation of energy). The system is conservative if the Hamiltonian $H(\boldsymbol{x})$ is bounded from below.

Energy losses (due to e.g. friction) are naturally included by terminating (some of) the ports with dissipative relationships. Indeed, consider instead of $\boldsymbol{G}(\boldsymbol{x}) \boldsymbol{u}$ in (18) a term

$$
\left[\begin{array}{ll}
\boldsymbol{G}(\boldsymbol{x}) & \boldsymbol{G}_{D}(\boldsymbol{x})
\end{array}\right]\left[\begin{array}{c}
\boldsymbol{u} \\
\boldsymbol{u}_{D}
\end{array}\right]=\boldsymbol{G}(\boldsymbol{x}) \boldsymbol{u}+\boldsymbol{G}_{D}(\boldsymbol{x}) \boldsymbol{u}_{D}
$$

and extend the corresponding output equations $\boldsymbol{y}=$ $\boldsymbol{G}^{T}(\boldsymbol{x}) \frac{\partial H}{\partial \boldsymbol{x}}(\boldsymbol{x})$ to

$$
\left[\begin{array}{c}
\boldsymbol{y} \\
\boldsymbol{y}_{D}
\end{array}\right]=\left[\begin{array}{l}
\boldsymbol{G}^{T}(\boldsymbol{x}) \frac{\partial H}{\partial \boldsymbol{x}}(\boldsymbol{x}) \\
\boldsymbol{G}_{D}^{T}(\boldsymbol{x}) \frac{\partial H}{\partial \boldsymbol{x}}(\boldsymbol{x})
\end{array}\right]
$$

Here $\boldsymbol{u}_{D}, \boldsymbol{y}_{D} \in \mathbb{R}^{m_{D}}$ denote the port variables which are terminated by static relationships of the form

$$
\boldsymbol{u}_{D}=-\boldsymbol{F}_{D}\left(\boldsymbol{y}_{D}\right)
$$

where the dissipative characteristics $\boldsymbol{F}_{D}: \mathbb{R}^{m_{D}} \rightarrow \mathbb{R}^{m_{D}}$ satisfy $\boldsymbol{y}_{D}^{T} \boldsymbol{F}_{D}\left(\boldsymbol{y}_{D}\right) \geq 0$. Hence (19) extends to

$$
\begin{aligned}
& H\left(\boldsymbol{x}\left(t_{1}\right)\right)-H\left(\boldsymbol{x}\left(t_{0}\right)\right)= \\
& \int_{t_{0}}^{t_{1}} \boldsymbol{u}^{T}(t) \boldsymbol{y}(t) \mathrm{d} t-\int_{t_{0}}^{t_{1}} \boldsymbol{y}_{D}^{T}(t) \boldsymbol{F}_{D}\left(\boldsymbol{y}_{D}(t)\right) \mathrm{d} t,
\end{aligned}
$$

expressing that the increase of internal energy in the system is equal to the externally supplied work minus the dissipated energy. The system is passive if the Hamiltonian $H(\boldsymbol{x})$ is bounded from below.

A particular case, but often used, is when (20) is linear, i.e., $\boldsymbol{u}_{D}=-\boldsymbol{R}_{D} \boldsymbol{y}_{D}$, where $\boldsymbol{R}_{D}$ is a symmetric and positive semi-definite matrix of appropriate dimensions. Then, (18) take the form

$$
\begin{aligned}
\dot{\boldsymbol{x}} & =(\boldsymbol{J}(\boldsymbol{x})-\boldsymbol{R}(\boldsymbol{x})) \frac{\partial H}{\partial \boldsymbol{x}}(\boldsymbol{x})+\boldsymbol{G}(\boldsymbol{x}) \boldsymbol{u}, \\
\boldsymbol{y} & =\boldsymbol{G}^{T}(\boldsymbol{x}) \frac{\partial H}{\partial \boldsymbol{x}}(\boldsymbol{x}),
\end{aligned}
$$

where $\boldsymbol{R}(\boldsymbol{x}):=\boldsymbol{G}_{D}(\boldsymbol{x}) \boldsymbol{R}_{D} \boldsymbol{G}_{D}^{T}(\boldsymbol{x})$. Equation (22) is called a port-Hamiltonian system with dissipation.

It will be shown next that (18) can be naturally accommodated to include systems with mass depending explicitly on position.

\subsection{Position-Dependent Models}

For a system consisting of $N$ particles with mass depending explicitly on position the kinetic (co-)energy takes the form $^{1}$

$$
T^{*}(\boldsymbol{r}, \dot{\boldsymbol{r}})=\frac{1}{2} \sum_{k=1}^{N} m_{k}\left(\boldsymbol{r}_{k}\right)\left\|\dot{\boldsymbol{r}}_{k}\right\|^{2} .
$$

Assuming for simplicity that there are no holonomic constraints $(\boldsymbol{r}=\boldsymbol{q})$, the associated conjugate momenta are given by

$$
\boldsymbol{p}_{k}=\frac{\partial T^{*}}{\partial \dot{\boldsymbol{r}}_{k}}(\boldsymbol{r}, \dot{\boldsymbol{r}})=m_{k}\left(\boldsymbol{r}_{k}\right) \dot{\boldsymbol{r}}_{k}, \quad k=1, \ldots, N .
$$

so that the kinetic energy yields

$$
T(\boldsymbol{r}, \boldsymbol{p})=\frac{1}{2} \sum_{k=1}^{N} m_{k}^{-1}\left(\boldsymbol{r}_{k}\right)\left\|\boldsymbol{p}_{k}\right\|^{2} .
$$

Let the Hamiltonian $H(\boldsymbol{r}, \boldsymbol{p})=T(\boldsymbol{r}, \boldsymbol{p})+V(\boldsymbol{r})$ represent the total stored energy, then the equations of motion for the $k$-th particle in port-Hamiltonian form read

$$
\begin{aligned}
\dot{\boldsymbol{x}}_{k} & =\boldsymbol{J}_{k} \frac{\partial H}{\partial \boldsymbol{x}_{k}}(\boldsymbol{x})+\boldsymbol{G}_{k} \boldsymbol{u}_{k}+\boldsymbol{G}_{D_{k}} \boldsymbol{u}_{D_{k}}, \\
\boldsymbol{y}_{k} & =\boldsymbol{G}_{k}^{T} \frac{\partial H}{\partial \boldsymbol{x}_{k}}(\boldsymbol{x}), \\
\boldsymbol{y}_{D_{k}} & =\boldsymbol{G}_{D_{k}}^{T} \frac{\partial H}{\partial \boldsymbol{x}_{k}}(\boldsymbol{x}),
\end{aligned}
$$

with $\boldsymbol{x}_{k}=\operatorname{col}\left(\boldsymbol{r}_{k}, \boldsymbol{p}_{k}\right)$,

$$
\boldsymbol{G}_{k}=\left[\begin{array}{c}
\mathbf{0} \\
\boldsymbol{B}_{k}
\end{array}\right], \quad \boldsymbol{J}_{k}=\left[\begin{array}{cc}
\mathbf{0} & \boldsymbol{I} \\
-\boldsymbol{I} & \mathbf{0}
\end{array}\right] .
$$

Inspired by the energy-balance in (17), let us instead of the static relationships (20) terminate the ports by

$$
\boldsymbol{u}_{D_{k}}=-\frac{1}{2} \dot{m}_{k}\left(\boldsymbol{r}_{k}\right) \boldsymbol{y}_{D_{k}} .
$$

Then, by letting

$$
\boldsymbol{G}_{D_{k}}=\left[\begin{array}{cc}
\mathbf{0} & \mathbf{0} \\
\mathbf{0} & \boldsymbol{I}
\end{array}\right]
$$

the port-Hamiltonian structure of a system with positiondependent mass is described by

$$
\begin{aligned}
{\left[\begin{array}{l}
\dot{\boldsymbol{r}}_{k} \\
\dot{\boldsymbol{p}}_{k}
\end{array}\right]=\left[\begin{array}{cc}
\mathbf{0} & \boldsymbol{I} \\
-\boldsymbol{I} & -\boldsymbol{D}_{k}(\boldsymbol{r}, \boldsymbol{p})
\end{array}\right]\left[\begin{array}{l}
\frac{\partial H}{\partial \boldsymbol{r}_{k}}(\boldsymbol{r}, \boldsymbol{p}) \\
\frac{\partial H}{\partial \boldsymbol{p}_{k}}(\boldsymbol{r}, \boldsymbol{p})
\end{array}\right]+\left[\begin{array}{c}
\mathbf{0} \\
\boldsymbol{B}_{k}
\end{array}\right] \boldsymbol{u}_{k}, } \\
\boldsymbol{y}_{k}=\left[\begin{array}{ll}
\mathbf{0} & \boldsymbol{B}_{k}^{T}
\end{array}\right]\left[\begin{array}{l}
\frac{\partial H}{\partial \boldsymbol{r}_{k}}(\boldsymbol{r}, \boldsymbol{p}) \\
\frac{\partial H}{\partial \boldsymbol{p}_{k}}(\boldsymbol{r}, \boldsymbol{p})
\end{array}\right], \quad k=1, \ldots, N,
\end{aligned}
$$

\footnotetext{
1 It is important to realize that (23) differs from (1) in that the masses of the particles are now depending explicitly on position, i.e., $m_{k}=m_{k}\left(\boldsymbol{r}_{k}\right)$, whereas the dependence of (2) on the generalized position coordinates is only due to a change of coordinates (removal of the holonomic constraints).
} 
where

$$
\begin{aligned}
\boldsymbol{D}_{k}(\boldsymbol{r}, \boldsymbol{p}) & :=\frac{1}{2} \dot{m}_{k}\left(\boldsymbol{r}_{k}\right) \boldsymbol{I}=\frac{1}{2} \dot{\boldsymbol{r}}_{k}^{T} \frac{\partial m_{k}}{\partial \boldsymbol{r}_{k}}\left(\boldsymbol{r}_{k}\right) \boldsymbol{I} \\
& =\frac{1}{2} m_{k}^{-1}\left(\boldsymbol{r}_{k}\right) \boldsymbol{p}_{k}^{T} \frac{\partial m_{k}}{\partial \boldsymbol{r}_{k}}\left(\boldsymbol{r}_{k}\right) \boldsymbol{I}
\end{aligned}
$$

represents the gained or lost mass.

Note that $\boldsymbol{D}_{k}$ plays a role that is similar to the dissipative matrix $\boldsymbol{R}$ that represents the energy converted into heat in (22). Equation (27) still has a Hamiltonian form, however, the matrix $\boldsymbol{D}_{k}$ is not (positive) definite because it represents the mass lost or gained.

In order to show that (27) indeed provides the correct equations of motion, we notice that by Newton's second law we must have $\dot{\boldsymbol{p}}_{k}=-\frac{\partial V}{\partial \boldsymbol{r}_{k}}(\boldsymbol{r})+\boldsymbol{B}_{k} \boldsymbol{u}_{k}$, or equivalently,

$$
-\frac{\partial T}{\partial \boldsymbol{r}_{k}}(\boldsymbol{r}, \boldsymbol{p})-\boldsymbol{D}_{k}(\boldsymbol{r}, \boldsymbol{p}) \frac{\partial T}{\partial \boldsymbol{p}_{k}}(\boldsymbol{r}, \boldsymbol{p}) \equiv \mathbf{0},
$$

which, in turn, implies (28).

The energy-balance of the overall system reads

$$
\begin{aligned}
H\left(\boldsymbol{x}\left(t_{1}\right)\right)-H\left(\boldsymbol{x}\left(t_{0}\right)\right)=\int_{t_{0}}^{t_{1}} \sum_{k=1}^{N} \boldsymbol{u}_{k}^{T}(t) \boldsymbol{y}_{k}(t) \mathrm{d} t \\
-\int_{t_{0}}^{t_{1}} \sum_{k=1}^{N}\left[\frac{\partial H}{\partial \boldsymbol{p}_{k}}(\boldsymbol{x}(t))\right]^{T} \boldsymbol{D}_{k}(\boldsymbol{x}(t)) \frac{\partial H}{\partial \boldsymbol{p}_{k}}(\boldsymbol{x}(t)) \mathrm{d} t,
\end{aligned}
$$

which now expresses that the increase of internal energy in the system is equal to the externally supplied work plus or minus - depending on the sign of $\boldsymbol{D}_{k}$ - the energy associated to the gained or lost mass.

\section{THE CABLE-REEL SYSTEM REVISITED}

Let us return to the cable-reel system discussed in Section 2. First, the equations of motion for the reel and suspended part of the cable subsystems are expressed as a portHamiltonian system. Secondly, it is shown that the portHamiltonian framework allows the two subsystems to be interconnected in a concise and systematic manner.

\subsection{The Reel Subsystem}

As illustrated in Section 2.2, the reel with the corresponding wounded cable is a position-dependent mass system. Hence, applying the procedure outlined in the previous section, the reel subsystem can be represented in a portHamiltonian fashion as follows. Since $n=N=1$ (the system consist of only one particle with one degree of freedom), let $\boldsymbol{x}=\operatorname{col}\left(\theta_{r}, p_{r}\right), \boldsymbol{u}=\tau_{r}$, and $\boldsymbol{y}=\omega_{r}$. Hence, according to $(27)$, we have

$$
\begin{aligned}
{\left[\begin{array}{l}
\dot{\theta}_{r} \\
\dot{p}_{r}
\end{array}\right]=\left[\begin{array}{cc}
0 & 1 \\
-1 & -D_{r}\left(\theta_{r}, p_{r}\right)
\end{array}\right]\left[\begin{array}{l}
\frac{\partial H_{r}}{\partial \theta_{r}}\left(\theta_{r}, p_{r}\right) \\
\frac{\partial H_{r}}{\partial p_{r}}\left(\theta_{r}, p_{r}\right)
\end{array}\right]+\left[\begin{array}{l}
0 \\
1
\end{array}\right] \tau_{r} } \\
\omega_{r}=\left[\begin{array}{ll}
0 & 1
\end{array}\right]\left[\begin{array}{l}
\frac{\partial H_{r}}{\partial \theta_{r}}\left(\theta_{r}, p_{r}\right) \\
\frac{\partial H_{r}}{\partial p_{r}}\left(\theta_{r}, p_{r}\right)
\end{array}\right]
\end{aligned}
$$

with the same Hamiltonian as given in (13), and

$$
D_{r}\left(\theta_{r}, p_{r}\right):=\frac{1}{2} \dot{I}_{r}\left(\theta_{r}\right)=\frac{1}{2} \frac{\mathrm{d} I_{r}}{\mathrm{~d} \theta_{r}}\left(\theta_{r}\right) I_{r}^{-1}\left(\theta_{r}\right) p_{r} .
$$

The associated energy-balance takes the form

$$
\begin{aligned}
H_{r}\left(\theta_{r}\left(t_{1}\right),\right. & \left.p_{r}\left(t_{1}\right)\right)-H_{r}\left(\theta_{r}\left(t_{0}\right), p_{r}\left(t_{0}\right)\right)=\int_{t_{0}}^{t_{1}} \tau_{r}(t) \omega_{r}(t) \mathrm{d} t \\
& -\int_{t_{0}}^{t_{1}} D_{r}\left(\theta_{r}(t), p_{r}(t)\right)\left[\frac{\partial H_{r}}{\partial p_{r}}\left(\theta_{r}(t), p_{r}(t)\right)\right]^{2} \mathrm{~d} t,
\end{aligned}
$$

which, by noting that $\omega_{r}=I^{-1}\left(\theta_{r}\right) p_{r}$, precisely coincides with (17).

\subsection{The Cable Subsystem}

Similarly, the suspended part of the cable is also a positiondependent system with mass $m_{s}\left(q_{s}\right)=m_{c}+\mu q_{s}$. Following (27), with $v_{s}=\dot{q}_{s}, p_{s}=m_{s}\left(q_{s}\right) v_{s}$, and $f_{s}$ the external force in the transmission point, the port-Hamiltonian description is

$$
\begin{aligned}
{\left[\begin{array}{l}
\dot{q}_{s} \\
\dot{p}_{s}
\end{array}\right]=\left[\begin{array}{cc}
0 & 1 \\
-1 & -D_{s}\left(q_{s}, p_{s}\right)
\end{array}\right]\left[\begin{array}{l}
\frac{\partial H_{s}}{\partial q_{s}}\left(q_{s}, p_{s}\right) \\
\frac{\partial H_{s}}{\partial p_{s}}\left(q_{s}, p_{s}\right)
\end{array}\right]+\left[\begin{array}{l}
0 \\
1
\end{array}\right] f_{s}, } \\
v_{s}=\left[\begin{array}{ll}
0 & 1
\end{array}\right]\left[\begin{array}{l}
\frac{\partial H_{s}}{\partial q_{s}}\left(q_{s}, p_{s}\right) \\
\frac{\partial H_{s}}{\partial p_{s}}\left(q_{s}, p_{s}\right)
\end{array}\right],
\end{aligned}
$$

with

$$
D_{s}\left(q_{s}, p_{s}\right):=\frac{1}{2} \dot{m}_{s}\left(q_{s}\right)=\frac{1}{2} \frac{\mathrm{d} m_{s}}{\mathrm{~d} q_{s}}\left(q_{s}\right) m_{s}^{-1}\left(q_{s}\right) p_{s} .
$$

and $H_{s}\left(q_{s}, p_{s}\right)=\frac{1}{2} m_{s}^{-1}\left(q_{s}\right) p_{s}^{2}+V\left(q_{s}\right)$, where we recall that $V\left(q_{s}\right)$ denotes the potential energy, see (8), due to the gravitational force acting on the cable.

\subsection{The Overall System}

Linking the reel and the suspended cable subsystems is achieved by setting

$$
\begin{aligned}
& \tau_{r}=R f_{s}, \\
& v_{s}=-R \omega_{r} .
\end{aligned}
$$

This leads to the port-Hamiltonian system ${ }^{2}$

$$
\left[\begin{array}{c}
\dot{\theta}_{r} \\
\dot{p}_{r} \\
\dot{q}_{s} \\
\dot{p}_{s}
\end{array}\right]=\left[\begin{array}{cccc}
0 & 1 & 0 & 0 \\
-1 & -D_{r} & 0 & 0 \\
0 & 0 & 0 & 1 \\
0 & 0 & -1 & -D_{s}
\end{array}\right]\left[\begin{array}{l}
\frac{\partial H_{r}}{\partial q_{r}} \\
\frac{\partial H_{r}}{\partial p_{r}} \\
\frac{\partial H_{s}}{\partial q_{s}} \\
\frac{\partial H_{s}}{\partial p_{s}}
\end{array}\right]+\left[\begin{array}{c}
0 \\
1 \\
0 \\
-R^{-1}
\end{array}\right] \lambda
$$

together with

\footnotetext{
${ }^{2}$ For ease of presentation we omit the arguments.
} 


$$
0=\left[\begin{array}{llll}
0 & 1 & 0 & -R^{-1}
\end{array}\right]\left[\begin{array}{l}
\frac{\partial H_{r}}{\partial q_{r}} \\
\frac{\partial H_{r}}{\partial p_{r}} \\
\frac{\partial H_{s}}{\partial q_{s}} \\
\frac{\partial H_{s}}{\partial p_{s}}
\end{array}\right],
$$

where $\lambda=\tau_{r}=R f_{s}$ denotes the internal constraint force. Note that this particular interconnection results in $\boldsymbol{G}=0$, i.e., the interconnected system no longer has external ports. The constraint on the velocities, (35), is represented by $(37)$.

The internal constraint force can be eliminated as follows. Let $\boldsymbol{b}=\left[\begin{array}{llll}0 & 1 & 0 & -\frac{1}{R}\end{array}\right]^{T}$ and let $\boldsymbol{b}^{\perp}$ denote its full-rank left annihilator ${ }^{3}$, i.e.,

$$
\boldsymbol{b}^{\perp} \boldsymbol{b}=\mathbf{0} \Rightarrow \boldsymbol{b}^{\perp}=\left[\begin{array}{llll}
1 & 0 & 0 & 0 \\
0 & 1 & 0 & R \\
0 & 0 & 1 & 0
\end{array}\right] \text {. }
$$

Hence, premultiplying (36) with $\boldsymbol{b}^{\perp}$, and using the definitions in (31) and (33), yields

$$
\left[\begin{array}{c}
\dot{\theta}_{r} \\
\dot{p}_{r}+R \dot{p}_{s} \\
\dot{q}_{s}
\end{array}\right]=\left[\begin{array}{l}
\frac{\partial H_{r}}{\partial p_{r}}\left(\theta_{r}, p_{r}\right) \\
-R \frac{\partial V}{\partial q_{s}}\left(q_{s}\right) \\
\frac{\partial H_{s}}{\partial p_{s}}\left(q_{s}, p_{s}\right)
\end{array}\right] .
$$

From the constraint in $(35), \dot{q}_{s}=-R \dot{\theta}_{r}$, the $q_{s}$-dynamics are superfluous and can thus be disregarded. Finally, defining the total momentum $p_{t}:=p_{r}+R p_{s}$, the interconnected system reduces to

$$
\left[\begin{array}{l}
\dot{\theta}_{r} \\
\dot{p}_{t}
\end{array}\right]=\left[\begin{array}{cc}
0 & 1 \\
-1 & 0
\end{array}\right]\left[\begin{array}{l}
\frac{\partial H_{t}}{\partial \theta_{r}}\left(\theta_{r}, p_{t}\right) \\
\frac{\partial H_{t}}{\partial p_{t}}\left(\theta_{r}, p_{t}\right)
\end{array}\right],
$$

with Hamiltonian function

$$
H_{t}\left(\theta_{r}, p_{t}\right)=\frac{1}{2}\left(I_{o}+m_{c} R^{2}\right)^{-1} p_{t}^{2}+V\left(\theta_{r}\right) .
$$

The port-Hamiltonian equations (40), with $\omega_{r}=\left(I_{o}+\right.$ $\left.m_{c} R^{2}\right)^{-1} p_{t}$, precisely correspond to the equation of motion derived in Section 2.1 .

\section{CONCLUDING REMARKS}

We have presented the application of the port-Hamiltonian formalism to systems with mass explicitly dependent on position. The inclusion of mass transport in the portHamiltonian framework turns out to be almost as straightforward as the inclusion of dissipative elements. The difference is that in the latter case free energy is always converted into heat, while in the former energy can both be added or extracted from the system. Apart from providing a consistent modeling framework, several control design methodologies are available that exploit the energy and interconnection structure; see for instance (Duindam et al. (2009)). It is precisely in this context that a portHamiltonian description can be of added value.

\section{REFERENCES}

Abraham, R. and Marsden, J.E. (1978). Foundations of Mechanics. Benjamin/Cummings (2nd edition).

Crelling, E.B., Janssens, F., Poelaert, D., Steiner, W., and Troger, H. (1997). On balance and variational formulations of the equation of motion of a body deploying along a cable. Journal of Applied Mechanics, 64(1), 369-374.

Cveticanin, L. (1998). Dynamics of machines with variable mass. CRC Press.

Duindam, V., Macchelli, A., Stramigioli, S., and Bruyninckx, H. (eds.) (2009). Modeling and Control of Complex Physical Systems: The Port-Hamiltonian Approach. Springer.

Pesce, C.P. (2003). The application of Lagrange equations to mechanical systems with mass explicitly dependent on position. J. Appl. Mech., 70, 751-756.

Pesce, C.P., Tannuri, E.A., and Casetta, L. (2006). The Lagrange equations for systems with mass varying explicitly with position: Some applications to offshore engineering. J. Braz. Mech. Sci. $\mathcal{E}_{3}$ Eng., XXVIII(4), 751756.

Van der Schaft, A.J. (2000). $\quad \mathcal{L}_{2}$-Gain and Passivity Techniques in Nonlinear Control. Springer-Verlag.

Willems, J.C. (2007). The behavioral approach to open and interconnected systems. IEEE Control Systems Magazine, 27(6), 46-99.

\footnotetext{
${ }^{3}$ In general, a full-rank left annihilator of $\boldsymbol{b} \in \mathbb{R}^{n \times m}, \boldsymbol{b}^{\perp}$, implies: $\boldsymbol{b}^{\perp} \boldsymbol{b}=\mathbf{0}$ and $\operatorname{rank}\left(\boldsymbol{b}^{\perp}\right)=n-m$.
} 